\title{
A dangerous vitious circle of the neonatal brain in bilirubin- induced neurologic dysfunction (BIND)
}

\begin{abstract}
On the basis of abundant research data and hypotheses the BIND is a neurodegenerative disease (ND) of immature brain caused by accumulation of free copper ions, unconjugated bilirubin (UCB), and UCB-Cu complex (as prooxidant), respectively, in the basal ganglia (BG) and other parts of the central nervous system (CNS) relevant to BIND. The main comorbidity is the hemolysis of neonatal red blood cells. During this process a great amount of heavy metals (mainly iron and copper) are liberated and producing reactive oxygen species (ROS). These elements may circulate in the bloodstream, and can pass through the immature blood-brain-barrier (BBB), finding entrance into the CNS. In addition, ROS contribute to increased BBB permeability creating a dangerous vitious circle in the neonatal brain, especially in the basal ganglia (BG). The beneficial effects of D-Penicillamine (D-PA) in neonates based on the capability of this drug to alter the NO system, and it is a strong antioxidant. Low molecular weight disulfides are the major products of D-PA metabolism in humans. The oxidation of D-PA in vivo may also important in the mode of action of the drug through simultaneous reduction of the ROS and reactive nitrogen species (RNS). Consequently, D-PA fulfils the criteria of a hybrid drug in the neonatal period by its ability to modulate both oxidative stress and NO pathway, and can be a neuroprotective agent in the pathophysiology of neurologic dysfunction.
\end{abstract}

Keywords: copper toxicity, bilirubin induced neurologic dysfunction, oxidative stress, copper bilirubin complex, d-Penicillamine in the neonatal period
Volume 3 Issue 3 - 2017

György Balla, István Pataki, Lajos Lakatos

Correspondence: Lajos Lakatos, Faculty of Medicine, Department of Pediatrics, University of Debrecen, Hungary, Email lakatosl@kenezykorhaz.hu

Received: April 04, 2017 | Published: May II, 2017
Abbreviations: BG, basal ganglia; ROS, reactive oxygen species; BBB, blood brain barrier; D-PA, d-penicillamine; UCB, unconjugated bilirubin; ND, neurodegenerative disease; CNS, central nervous system; RNS, reactive nitrogen species

\section{Introduction}

The copper as a transitional metal is a powerful oxidant producing the highly damaging hydroxyl $\left(\mathrm{OH}^{-}\right)$radical. A literature review was aimed at assisting us (as paediatricians) to provide further understanding with bilateral symmetrical basal ganglia (BG) and thalamic lesions caused by Wilson's disease and BIND..$^{1,2}$ Elevated levels of "free" or loosely bound metal ions can exert toxic effects, and in order to maintain homeostatic levels to protect the brain (especially the basal ganglia/BG) and retinal cells from their toxicity. ${ }^{3}$ In fact that appropriate mechanisms exist such as metal transporters, chaperones, and the presence of certain storage molecules that tightly bind metals to form nontoxic products, however, these mechanisms do not work in the neonatal period. ${ }^{4}$

Very wide-ranging studies have long been made on the possible biochemical transformations of unconjugated bilirubin (UCB). Particular attention has been paid to its photochemical and redox reactions but the relevant publications comprise only a very small proportion of those dealing with the molecular biochemistry of UCB and metals interactions. Bilirubin has a special affinity for the globus pallidus, the hippocampus, and the subthalamic nucleus because they are also target brain regions for divalent metal $(\mathrm{Cu}, \mathrm{Fe}, \mathrm{Zn}$ etc.) accumulation. ${ }^{5}$

Our new concept addresses the medical necessity of chelation therapy (with D-Penicillamine-/D-PA) in the neonatal period. ${ }^{6}$
As it is feasible that UCB molecule possesses particular affinity to copper stored in BG where copper-bilirubin complex can be formed together with the production of hydroxyl radical. Therefore, BIND is a neurological damage of immature brain caused by accumulation of free metals and UCB-Cu complex (as prooxidant) in the BG and other parts of central nervous system relevant to BIND. The main comorbidity is the hemolysis. During this process a great amount of heavy metals (mainly iron and copper) are liberated and producing ROS. These elements may circulate in the bloodstream, and can pass through the immature BBB, finding entrance into the CNS. In addition, ROS contribute to increased BBB permeability creating a dangerous vitious circle in the neonatal brain, especially in the BG. In strictly biological terms the two most important such metals are iron and copper. ${ }^{7,8}$ The beneficial effects of D-PA in neonates based on the capability of this drug to alter the NO system, and it is a strong antioxidant. Low molecular weight disulfides are the major products of D-PA metabolism in humans. ${ }^{9}$ The oxidation of D-PA in vivo may also important in the mode of action of the drug through simultaneous reduction of the ROS and reactive nitrogen species (RNS). Consequently, D-PA fulfils the criteria of a hybrid drug in the neonatal period by its ability to modulate both oxidative stress and NO pathway, and can be a neuroprotective agent in the pathophysiology of neurologic dysfunction (Figure 1). ${ }^{10}$

We hope that our concept will help answer some of the unsolved questions and concerns occurred in the etiology and pathomechanisms of BIND and other neurodegenerative/neurodevelopment disorders. The beneficial neuropharmacological actions of metal-targeted (chelating) agents most likely arise from local metal redistribution rather than from massive metal removal. ${ }^{11,12}$ The chelation therapy for non-metal overload indications continues to be investigated. Our 
present articles address the medical necessity of the use of a chelating agent (D-PA) in the prevention or treatment of neonatal brain injuries.

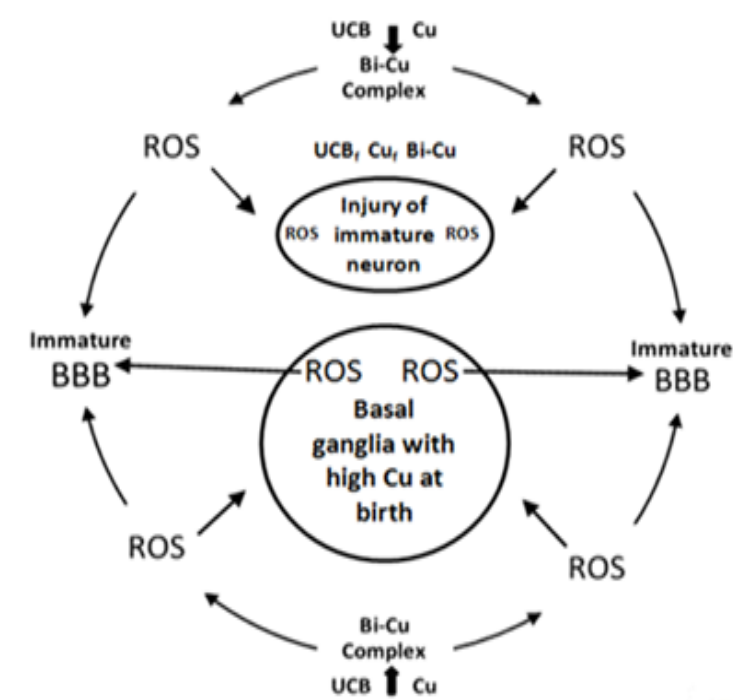

Figure I Molecular mechanism of BIND.

$\cup C B$, unconjugated bilirubin; $\mathrm{UCB}_{\mathrm{f}}$, free unconjugated bilirubin; $\mathrm{Cu}_{f}$, free $\mathrm{Cu}$; ROS, reactive oxygen species; $\mathrm{BBB}$, blood brain barrier

\section{Acknowledgements}

None.

\section{Conflict of interest}

The author declares no conflict of interest.

\section{References}

1. Johnson L, Bhutani VK. The Clinical Syndrome of Bilirubin-Induced Neurologic Dysfunction. Semin Perinatol. 2011;35(3):101-113.
2. Wennberg RP, Ahlfors CE, Bhutani VK, et al. Toward understanding kernicterus: A challenge to improve the management of jaundiced newborns. Pediatrics. 2006;117(2):474-485.

3. Bhutani V, Wong R. Bilirubin-induced neurologic dysfunction. 2015;20(1):1-64.

4. Jethani J, Marwah K, Nikul, et al. Ocular abnormalities in patients with beta thalassemia on transfusion and chelation therapy: Our experience. Indian J Ophthalmol. 2010;58(5):451-452.

5. Bolognin S, Messori L, Zatta P. Metal ion Physiopathology in Neurodegenerative Disorder. Neuromolecular Med. 2009;11(4):223-238.

6. György Balla, Lajos Lakatos, Zsuzsanna Vekerdy Nagy. Chelation therapy in the neonatal period: D-Penicillamine can exert neuroprotective effects in kernicterus and retinopathy of prematurity. IJPSR. 2015;6(10):4269-4276.

7. Adhikari S, Joshi R, Gopinathan C. Bilirubin as an antiprecipitant against copper mediated denaturation of bovine serum albumin: formation of copper-bilirubin complex. Biochim Biophys Acta. 1998;1380(1):109114.

8. Adhikari S. Personal communication. Hungary; 2016.

9. Joyce DA, Day RO. D-penicillamine and D-penicillamine-protein disulphide in plasma and synovial fluid of patients with rheumatoid arthritis. Br J Clin Pharmacol. 1990;30(4):511-517.

10. Rahimi N, Sadeghzadeh M, Javadi Paydar M, et al. Effects of D-penicillamine on pentylenetetrazole-induced seizures in mice: Involvement of nitric oxide/NMD pathways. Epilepsy Behav. 2014;39(2):42-47.

11. Mot AI, Wedd AG, Sinclair L, et al. Metal attenuating therapies in neurodegenerative disease. Expert Rev Neurother. 2011;11(12):1717-1745.

12. Jellinger KA. Chapter One-The Relevance of Metals in the Pathophysiology of Neurodegeneration, Pathological Considerations. Int Rev Neurobiol. 2013;110(1):1-47. 